\section{Determinación de los parámetros de transferencia de calor durante la cocción de bollos de maíz amarillo}

\section{Determination of heat transference parameters during the cooking of yellow corn muffins (bollos de maiz Amarillo)}

\author{
José David Torres-González*, Luis Alberto Gallo-García **, Diofanor Acevedo- \\ Correa***
}

*Estudiante de Doctorado en Ingeniería Química y Bioprocesos, MSc Ciencias Agroalimentaria. Ingeniero de Alimentos, Universidad de Cartagena, Avenida el Consulado, Calle 30 No. 48-152. Cartagena-Colombia. **Ingeniero de Alimentos. Universidad de Cartagena, Facultad de Ingeniería, Cartagena-Colombia. ***Doctor en Ingeniería. Especialista en Ciencia y Tecnología de Alimentos. Docente Universidad de Cartagena.

\section{Resumen}

Los bollos son alimentos autóctonos elaborados a base de masa de maíz, estos se envuelven en las hojas de la mazorca y se cocinan por ebullición. En esta investigación se determinaron los parámetros de la transferencia de calor durante la cocción de bollos de maíz de la variedad cariaco amarillo. El proceso se realizó en un sistema automatizado con el control de la temperatura y el tiempo. Las propiedades termofísicas se calcularon en función de la composición química y la temperatura. El coeficiente de transferencia de calor se calculó mediante regresión lineal a partir de los datos de relación adimensional de temperatura vs al tiempo. Los resultados indicaron que el centro térmico del producto llegó a $75 \pm 1^{\circ} \mathrm{C}$ en $25 \mathrm{minutos}$. La composición del bollo cocido fue (humedad $=29.54 \pm 1.07$, grasa $=6.51 \pm 0.49$, cenizas $=0.48 \pm 0.05$, proteínas $=7.59 \pm 0.29$, fibra $=9.65 \pm 0.77$, carbohidratos $=45.89 \pm 3.22$ y calorías $=373.36 \pm 9.36 \mathrm{kcal} / 100 \mathrm{~g}$ ). Los coeficientes de conductividad y difusividad térmica aumentaron significativamente con la temperatura de procesamiento, obteniéndose valores máximos en el producto final de $0.68 \pm 0.07 \mathrm{~W} / \mathrm{m}^{\circ} \mathrm{C}$ y $1.98 \pm 0.02 \times 10-7 \mathrm{~m} 2 / \mathrm{s}$. La capacidad calorífica y la densidad en el producto cocido fueron de $3145.36 \pm 88.45 \mathrm{~J} / \mathrm{kg}{ }^{\circ} \mathrm{C}$ y $1254.49 \pm 30.47$ respectivamente. El coeficiente convectivo fue de $118.29 \mathrm{~W} /$ $\mathrm{m} 2{ }^{\circ} \mathrm{C}$, el cual estuvo dentro del rango reportado para productos amiláceos similares. El entendimiento de los parámetros de transferencia de calor durante la cocción de bollo de maíz es importante para la optimización de procesos térmicos aplicados en este alimento.

Palabras clave: caloría, fibra, nutrición, transferencia de calor vegetal,

\begin{abstract}
Corn muffins (bollos) are indigenous food stuffs, prepared from corn dough, wrapped in cob leaves, and cooked by ebullition. This research determined heat transference parameters during corn muffin cooking, yellow cariaco variety. The process was performed in an automated system, with temperature and time control. Thermophysical properties were calculated in function of chemical composition and temperature. Heat transference coefficient was calculated through linear regression, parting from data of dimensionless relationship of temperature vs time. Results showed that thermal center of the product reached $75 \pm 1^{\circ} \mathrm{C}$ in 25 minutes. Composition of cooked corn muffin (bollo cocido) was (humidity $=29.54+1.07$; fat $=6.51+0.49 ;$ ash $=0.48+0.05 ;$ proteins $=7.59+0.29$; fiber $=9.65+0.77 ;$ carbohydrates $=45.89+3.22 ;$ and calories $=$ $373.36+9.36 \mathrm{kcal} / 100 \mathrm{~g}$ ). Conductivity and thermal diffusivity coefficients meaningfully increased as a result of processing temperature. Maximum values in the final product of $0.68+0.07 \mathrm{~W} / \mathrm{m}{ }^{\circ} \mathrm{C}$ and $.98 \pm 0.02 \times 10-7 \mathrm{~m} 2 / \mathrm{s}$. Heat capacity and density of cooked product were $3145.36 \pm 88.45 \mathrm{~J} / \mathrm{kg}{ }^{\circ} \mathrm{C}$ and $1254.49 \pm 30.47$ respectively. Convective coefficient was $118.29 \mathrm{~W} / \mathrm{m} 2{ }^{\circ} \mathrm{C}$, it was within the range reported for similar starchy products. Understanding heat transference parameters during cooking corn muffins is important for optimization of thermal processes, applied to this kind of food.
\end{abstract}

Key words: Heat transference, nutrition, calorie, vegetal, fiber.

Marco de referencia para la implementación del mapa de ruta establecido en los lineamientos nacionales de apertura de datos del sector público y su integración con procesos de descubrimiento de conocimiento e inteligencia de negocios. Grupo de investigación GITIR, Universidad de Caldas
Recibido: 20/04/2016

Revisado: 07/06/2016

Aceptado: 01/12/2016

Correspondencia de autor:

jtorresg3@unicartagena.edu.co

(C) 2016 Universidad La Gran Colombia. Este es un artículo de acceso abierto, distribuido bajo los términos de la licencia Creative Commons Attribution License, que permite el uso ilimitado, distribución y reproducción en cualquier medio, siempre que el autor original y la fuente se acrediten.

Cómo citar:

Torres-Gonzáles, J.D., Gallo-Garcia, L.A., Acevedo, D. (2016) Determinación de los parámetros de tranferencia de calor durante la cocción de bollos de maiz amarillo. UGCiencia 22, 213-225.

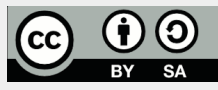




\section{Introducción}

El maíz (Zea mays L.) constituye uno de los principales cereales cultivados a nivel mundial, debido a su amplio consumo y utilización en la dieta humana para la elaboración de diversos productos (Gamboa, García y Tablante, 2012). Este aporta proteínas, lípidos y poca agua, es rico en hidratos de carbono, vitaminas $\mathrm{A}$, B1, B2, B3, B6, B9, E y C y en sales minerales como potasio, magnesio, hierro, calcio, zinc, sodio y fósforo. El germen del grano posee un aceite rico en ácidos grasos insaturados, además tiene bajo contenido de calcio y elevado de fósforo. Se ha señalado que es una buena fuente de fibra de ambos tipos, soluble e insoluble por lo que se aconseja su consumo en caso de estreñimiento, niveles elevados de colesterol y triglicéridos en la sangre (Arámbula-Villa, Barron-Avila, Gonzalez-Hernández, Moreno-Martinez y Luna-Barcenas, 2001).

El bollo de maíz es uno de los principales alimentos en la gastronomía típica de la región Caribe Colombiana, por sus características de composición, ha sido comúnmente catalogado como de fácil deterioro (Torres, Acevedo y Montero, 2016a). Durante el proceso de elaboración, la masa preparada se envuelve en hojas de maíz y se somete a cocción por ebullición, siendo esta etapa la más crítica dado que sus características físicas y organolépticas cambian (Torres, Acevedo y Tirado, 2015). Este producto normalmente es comercializado de forma tradicional en las calles, plazas públicas y en restaurantes, pero debido a la alta demanda y consumo en los últimos años, se ha empezado a preparar a escala industrial (Castilla, Mercado, Mendoza y Monroy, 2011; Torrenegra et al., 2013).

La cocción puede ser definida como el aumento de la temperatura de los alimentos en un medio de procesamiento adecuado, durante un tiempo suficiente para transformar su microestructura y desarrollar características de calidad especiales que los hagan más apetecibles por parte de los consumidores (Siripon, Tansakul y Mittal, 2007; Palermo, Pellegrini y Fogliano, 2014). Se ha señalado que cada alimento tiene un tiempo específico de cocción, el cual depende de la velocidad de transmisión de calor, el grado de hidratación, porosidad, espesor y composición química (Carciofi, Faistel, Aragão y Laurindo, 2002; Ayadi, Makni y Attia, 2009). También se ha indicado que durante este proceso térmico, se consigue la destrucción de toxinas e inhibición algunos de los agentes microbianos patógenos que se encuentran comúnmente en los alimentos crudos, por ello la importancia de controlar este tratamiento térmico (Murador, Mercadante y de Rosso, 2016). Se ha indicado que en los productos amiláceos durante la cocción en agua, se destruyen las estructuras rígidas de los tejidos y se gelatinizan los almidones, con lo que se logra el ablandamiento superficial y una mayor digestibilidad (Iborra $\square$ Bernad, García $\square$ Segovia y Martínez $\square$ Monzó, 2014). Las propiedades termofísicas son parámetros importantes en la descripción de la transferencia de calor durante la cocción, ya que por medio de éstas es posible conocer la estructura de los alimentos, suscitando grandes ventajas su comprensión especialmente por los costos energéticos, la eficiencia en el diseño de equipos y el aseguramiento de la calidad de los productos (Alvis, Caicedo y Peña, 2012; Alvis, González y Arrázola, 2015; Kanjanapongkul, 2017). En estas se incluyen, el calor específico, conductividad, difusividad térmica, que se relacionan a su vez con la densidad.

Actualmente existe mucha variación de las características físicas del bollo de maíz en los diferentes lugares donde se procesa, especialmente en la etapa de cocción, la cual requiere ser estandarizada para asegurar la calidad final del producto. Estos puntos son de vital importancia, ya que un tiempo prolongado representa un gasto energético excesivo, elevación en los costos de producción, deterioro en sus propiedades organolépticas y deficiencias en los contenidos nutricionales (Arámbula-Villa et al., 2001; Torres et al., 2015). Mientras que un tiempo demasiado corto, puede ocasionar que no se alcancen las temperaturas óptimas de cocción en el centro del alimento y originaría el rechazo por los consumidores, tal como se ha señalado en otras matrices amiláceas (Bouasla, Wójtowicz y Zidoune, 2017). La optimización del proceso de cocción en esta medida, resulta de vital importancia ya que permitiría reducir el coste de la energía utilizada y ayudaría a incrementar la aceptabilidad y calidad del producto en los lugares de producción y comercialización. Teniendo en cuenta lo anterior, el objetivo de esta investigación fue determinar los parámetros de transferencia de calor del bollo de maíz de la variedad cariaco amarillo durante el proceso de cocción por ebullición. 


\section{Materiales y métodos}

\section{Proceso de elaboración del producto}

Para la elaboración de los bollos de maíz, se utilizaron las técnicas comunes de los vendedores del producto en la ciudad de Cartagena (Torrenegra et al., 2013; Torres et al., 2015). Fueron obtenidos unos $50 \mathrm{~kg}$ de maíz de la variedad cariaco amarillo, el cual fue escogido de un centro de abastos, teniendo en cuenta el tamaño de las mazorcas mayor a $30 \mathrm{~cm}$. La materia prima fue trasladada en recipientes asépticos hasta las instalaciones de los laboratorios del grupo de Investigación NUSCA de la Universidad de Cartagena, donde se les quitó manualmente las hojas de la mazorca, las cuales se sumergieron en una solución de hipoclorito de sodio con una concentración base de $5 \mathrm{mg} / \mathrm{L}$ y posteriormente se usaron para la envoltura de los bollos. Los granos obtenidos se pesaron y rápidamente se llevaron a un molino industrial marca corona ${ }^{\circledR}$, donde se transformó en una masa suave. Se obtuvo un rendimiento de $60 \%$ respecto a la cantidad de maíz base. A la masa se le adicionaron todos los ingredientes requeridos: cloruro de sodio (10 g/ $\mathrm{kg}$ de masa), bicarbonato de sodio ( $5 \mathrm{~g} / \mathrm{kg}$ de masa) y azúcar ( $8 \mathrm{~g} / \mathrm{kg}$ de masa) estos fueron mezclados completamente. La cantidad medida de $160 \mathrm{~g}$ de masa se envolvió en su empaque tradicional (hojas de la mazorca e hilo) y enseguida fueron llevados al recipiente metálico de cocción a una temperatura base de $98 \pm 1{ }^{\circ} \mathrm{C}$. Después de esto las muestras se retiraron del agua y se ubicaron en un cestillo de malla metálica con perforaciones las cuales facilitaron el escurrido. A los 5 minutos de enfriamiento se almacenaron en bolsas de polietileno de alta densidad a temperatura de refrigeración de $15{ }^{\circ} \mathrm{C}$, antes de los respectivos análisis.

\section{Procedimiento de cocción}

La cocción se realizó en un baño de agua a temperatura controlada, el cual se diseñó para este fin en acero inoxidable tipo 304 y teniendo en cuenta la normativa colombiana (Torres, Acevedo y Montero, 2016b). Fueron utilizados termopares tipo J para determinar el historial de temperatura de los bollos de maíz cada 5 minutos. Estos fueron ubicados en el centro geométrico del producto $\left(\mathrm{T}_{\mathrm{c}}\right)$, en la superficie a una profundidad de $0.5 \mathrm{~mm}\left(\mathrm{~T}_{\mathrm{i}}\right)$ y en el medio de calentamiento $\left(\mathrm{T}_{\infty}\right)$. La relación muestra-agua durante la cocción fue 1:6 (160 g/1000 g de agua). Cuando la temperatura central llegó a $75^{\circ} \mathrm{C}$ el proceso fue terminado. En la figura 1, se esquematiza la orientación del producto el cual poseía una longitud de $20 \mathrm{~cm}$ y un diámetro de $6 \mathrm{~cm}$.

Figura 1. Esquema de la máquina para la cocción atmosférica del bollo de maíz de geometría cilíndrica. Fuente:

Torres et al. (2016b)

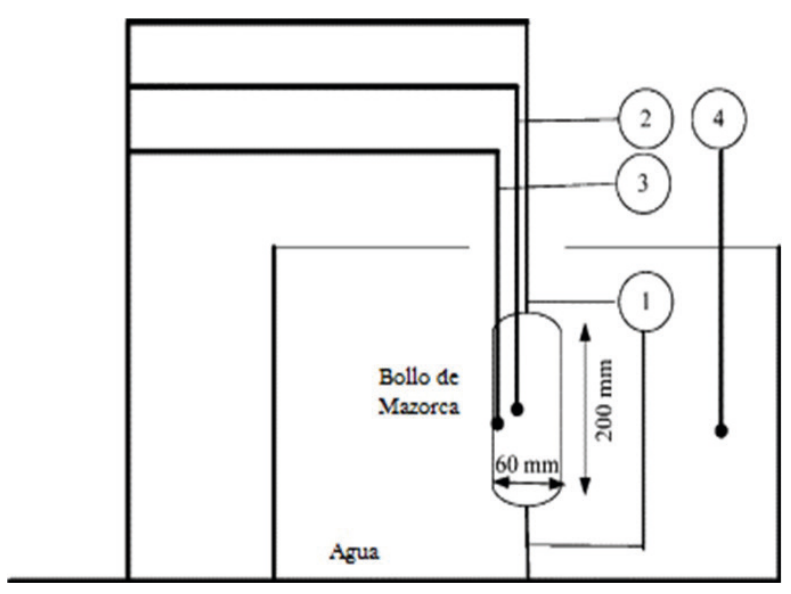

(a) Esquema de cocción: (1) barras que sostiene al bollo, (2) termopar central, (3) termopar superficial, (4) termopar en el agua.

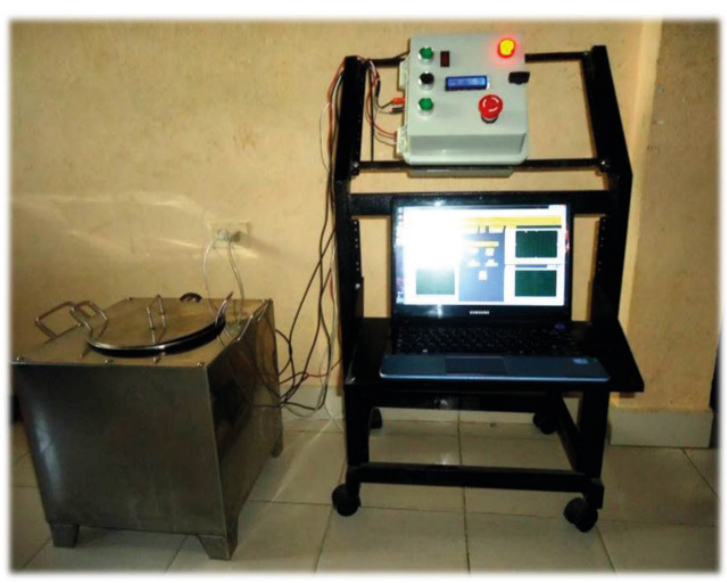

(b) Equipo de cocción encendido conectado a un computador portátil. 


\section{Análisis bromatológicos}

Las mediciones se realizaron a los bollos de maíz y se compararon los datos con los obtenidos de las masas antes de la cocción. Las muestras sólidas se maceraron y homogenizaron dentro de bolsas ziploc de autocierre, inmediatamente se realizaron las siguientes determinaciones, siguiendo la metodología de la AOAC (2012) para cereales y derivados: $\%$ humedad por secado en horno convector LT04/5 hasta peso constante a $105^{\circ} \mathrm{C}$ (Método, 925.09); grasa por extracción con éter de petróleo en el equipo Soxhlet (Método, 945.38); Proteinas por digestión ácida con el método Kjeldahl utilizando un factor de conversión de nitrógeno N x 6.25 (Método, 920.87). Cenizas por incineración total a $550^{\circ} \mathrm{C}$ (Método, 923.03). Fibra cruda utilizando el equipo determinador (TE-149 TECNAL) con el secado previo de la muestra, incineración durante 90 minutos y posterior enfriamiento en el desecador (Método, 962.09). Una vez obtenidos estos porcentajes, la diferencia de 100 se utilizó para hallar los carbohidratos totales, ecuación (1). Y Las calorías (kcal/100 g) se determinaron mediante la relación empírica que se enmarca en la ecuación (2) (Torres et al., 2016a). Todos los datos se expresaron como la media con su respectiva desviación estándar.

\section{Determinación de las propiedades termofísicas}

El calor específico $(C p)$, la densidad $(\rho)$, conductividad $(\mathrm{k})$ y la difusividad térmica $(\alpha)$ de las muestras enteras de bollos de maíz, se determinaron utilizando las fórmulas sugeridas en la tabla 1, con base en la composición del producto y la temperatura de procesamiento. Estos modelos fueron sistematizados en el programa computacional DEPROTER (Determinación de Propiedades Termofisicas) desarrollado por Alvis, Caicedo y Peña (2012). Los cálculos se realizaron por triplicado y los resultados se expresaron como la media con su desviación estándar.

Tabla 1. Modelos utilizados para calcular las propiedades termofísicas de las arepas con huevo

\begin{tabular}{|c|c|c|c|c|}
\hline Propiedad & Unidades & Modelos* & $\mathbf{R}^{2}$ & Referencia \\
\hline \multirow{2}{*}{$\begin{array}{l}\text { Conducti- } \\
\text { vidad tér- } \\
\text { mica }(k)\end{array}$} & \multirow{2}{*}{$\mathrm{W} / \mathrm{m}^{\circ} \mathrm{C}$} & $\begin{array}{l}\mathrm{k}=0.25 \mathrm{X}_{\mathrm{HC}}+0.16 \mathrm{X}_{\mathrm{P}}+0.16 \mathrm{X}_{\mathrm{GR}} \\
+0.14 \mathrm{X}_{\mathrm{CZ}}+0.48 \mathrm{X}_{\mathrm{H} 2 \mathrm{O}}\end{array}$ & 0.92 & \multirow{8}{*}{$\begin{array}{l}\text { Alvis et al. } \\
\quad(2012)\end{array}$} \\
\hline & & $\begin{array}{l}\mathrm{k}=0.16+0,02 \mathrm{~T}+0.29 \mathrm{X}_{\mathrm{H} 2 \mathrm{O}}+3.48 \\
\mathrm{x} 10^{-7} \mathrm{~T}^{2}\end{array}$ & 0.95 & \\
\hline \multirow{2}{*}{$\begin{array}{l}\text { Calor espe- } \\
\text { cífico }(C p)\end{array}$} & \multirow{2}{*}{$\mathrm{kJ} / \mathrm{kg}{ }^{\circ} \mathrm{C}$} & $\begin{array}{l}\mathrm{C}_{\mathrm{p}}=1.42 \mathrm{X}_{\mathrm{HC}}+1.55 \mathrm{X}_{\mathrm{p}}+1.68 \\
\mathrm{X}_{\mathrm{GR}}+0.85 \mathrm{X}_{\mathrm{CZ}}+4.18 \mathrm{X}_{\mathrm{H} 2 \mathrm{O}}\end{array}$ & 0.91 & \\
\hline & & $\begin{array}{l}\mathrm{C}_{\mathrm{p}}=489.98+3313 \mathrm{X}_{\mathrm{H} 2 \mathrm{O}}+24 \mathrm{~T}- \\
53 \mathrm{X}_{\mathrm{H} 2 \mathrm{O}} * \mathrm{~T}+33.7 \mathrm{X}_{\mathrm{H} 2 \mathrm{O}}{ }^{2} \mathrm{~T}\end{array}$ & 0.96 & \\
\hline \multirow{2}{*}{$\begin{array}{l}\text { Densidad } \\
(\rho)\end{array}$} & \multirow[t]{2}{*}{$\mathrm{kg} / \mathrm{m}^{3}$} & $\begin{array}{l}\rho=1.34 \mathrm{X}_{\mathrm{HC}}+1.15 \mathrm{X}_{\mathrm{P}}+0.75 \mathrm{X}_{\mathrm{GR}} \\
+1.95 \mathrm{X}_{\mathrm{CZ}}+0.95 \mathrm{X}_{\mathrm{H} 2 \mathrm{O}}\end{array}$ & 0.94 & \\
\hline & & $\rho=1553-568,81 X_{\mathrm{H} 2 \mathrm{O}}$ & 0.93 & \\
\hline \multirow[t]{2}{*}{$\begin{array}{l}\text { Difusividad } \\
\text { térmica }(\alpha)\end{array}$} & \multirow[t]{2}{*}{$10^{-7} \mathrm{~m}^{2} / \mathrm{s}$} & $\begin{array}{l}\alpha=\left(0.08-0.06 \mathrm{X}_{\mathrm{H} 2 \mathrm{O}}+0.12 \mathrm{X}_{\mathrm{H} 2 \mathrm{O}}\right. \\
{ }^{2}+6.87 \times 10^{-4} \mathrm{X}_{\mathrm{H} 2 \mathrm{O}}{ }^{2} \mathrm{~T}-5.17 \times 10-4 \\
\left.\mathrm{X}_{\mathrm{H} 2 \mathrm{O}}{ }^{2} \mathrm{~T}^{2}\right) \times 10^{-6}\end{array}$ & 0.97 & \\
\hline & & $\alpha=\mathrm{k} / \rho^{*} \mathrm{Cp}$ & 0.85 & \\
\hline
\end{tabular}

*Dónde: $\mathrm{X}_{\mathrm{HC}}, \mathrm{X}_{\mathrm{P}} \mathrm{X}_{\mathrm{GR}}, \mathrm{X}_{\mathrm{CZ}} \mathrm{y}_{\mathrm{H} 2 \mathrm{O}}$ representan las fracciones másicas de carbohidratos, proteínas, grasas, cenizas y humedad del producto. Los valores estuvieron dados para un rango de temperaturas de -4 a $200^{\circ} \mathrm{C}$. Fuente: Alvis $e t$ al. (2012) 


\section{Determinación del coeficiente convectivo de transferencia de calor (h)}

El coeficiente $(h)$ no es una propiedad de los alimentos, pero es un parámetro importante y necesario para el diseño, control y modelamiento de los sistemas de calentamiento. El modelo elegido para calcular $h$ durante el proceso de cocción del bollo de maíz, se basó en el análisis de los sistemas concentrados, el cual ha sido aplicado con éxito por otros autores en procesos térmicos similares (Vélez-Ruiz y Sosa-Morales, 2003; Mondal y Dash, 2016), debido a su sencillez y buen ajuste con los datos experimentales $\left(\mathrm{R}^{2}>0.90\right)$. En esta investigación se asumieron los siguientes supuestos: el producto fue homogéneo e isótropo, el calor se desplazó en dirección radial, la envoltura del producto no afectó el valor de las propiedades termofísicas, siendo estas constantes. Así mismo, la distribución del contenido de humedad fue uniforme y no hubo producción de calor interno. Al hacer un balance energético entre el fluido y el sólido se obtuvo que: (El incremento de la energía interna del cuerpo durante $d t)=($ Calor que se desplazó hacia el cuerpo durante $d t$ ) como se muestra en la ecuación (3):

$$
\dot{Q}_{\text {conducción }}=\dot{Q}_{\text {convección }}
$$

Dónde la convección fue descrita mediante la ley de enfriamiento de Newton, como se presenta en la ecuación (4):

$$
\dot{Q}=h A\left[T-T_{\infty}\right] d t
$$

En esta la temperatura del fluido fue mayor que la del producto $\mathrm{T} \infty>\mathrm{Ti}$ (calentamiento); $h$ es coeficiente de transferencia de calor; A es el área superficial. Por lo tanto el balance de calor se expresó en la ecuación (5):

$$
\dot{m} \Delta h=-h A\left[T-T_{\infty}\right] d t
$$

El cambio en la entalpia fue , Cp. capacidad calorífica $\left(\mathrm{J} / \mathrm{kg}^{\circ} \mathrm{C}\right)$. Llegando a la ecuación (6):

$$
\rho V C p d T=-h A\left[T-T_{\infty}\right] d t
$$

Al separar variables, teniendo en cuenta que y estableciendo los límites de integración se obtuvo la ecuación (7):

$$
\int_{T_{i}}^{T_{(t)}} \frac{d\left[T-T_{\infty}\right]}{T-T \infty}=-\frac{h A}{\rho C p V} \int_{0}^{t} d t
$$

Al resolver la integral se consiguió la ecuación (8):

$$
\operatorname{Ln}\left[\frac{T(t)-T \infty}{T i-T \infty}\right]=-\frac{h A}{\rho C p V} t
$$

Para este caso, el bollo de maíz poseía con una geometría cilíndrica, por lo tanto, el área total se calculó sumando el área lateral más el área de sus dos bases como: $A=2 \pi r L+2 \pi r^{2}$, mientras que el volumen se halló como: $V=\pi r^{2} L$. Al graficar, la pendiente de la sección lineal (-m) de esta gráfica se igualó a , y conocidos los demás valores se calculó el coeficiente convectivo de transferencia de calor, mediante la ecuación (9). 


$$
h=\frac{m \rho V C p}{A}
$$

El número de biot de transferencia de calor se determinó aplicando la ecuación (10).

$$
\text { Biot }=\frac{h L}{k}
$$

Dónde:

$h=$ coeficiente convectivo en la superficie $\left(\mathrm{W} / \mathrm{m}^{2 \circ} \mathrm{C}\right)$.

$L=$ longitud característica en m, definida como el volumen dividido por el área (V/A).

$k=$ conductividad térmica del material $\left(\mathrm{W} / \mathrm{m}{ }^{\circ} \mathrm{C}\right)$.

\section{Análisis estadísticos}

Los análisis en los laboratorios bajo condiciones controladas de temperatura y humedad ambiente, se efectuaron por triplicado y los resultados se expresaron como la media con su respectiva desviación estándar. Se realizaron ANOVA de una vía y para comparar las medias de los tratamientos se utilizó el test HSD de Tukey con un nivel de significancia del $5 \%(\mathrm{p} \leq 0.05)$. El procesamiento de la información se realizó con el paquete estadístico comercial Statgrapichs Centurión 16.1.15 (Keygen, U.S.A) en un computador personal.

\section{Resultados}

En la tabla 2 se muestran los datos obtenidos de los análisis bromatológicos, realizados a la masa y al producto después del proceso de cocción.

\begin{tabular}{|c|c|c|c|c|c|c|c|}
\hline $\begin{array}{c}\text { Prueba/Mues- } \\
\text { tra* }\end{array}$ & $\begin{array}{c}\text { Grasa } \\
(\%)\end{array}$ & $\begin{array}{c}\text { Carbohidratos } \\
(\%)\end{array}$ & Humedad (\%) & $\begin{array}{c}\text { Fibra } \\
(\%)\end{array}$ & Cenizas $(\%)$ & Proteínas (\%) & $\begin{array}{c}\text { Calorías } \\
(\mathrm{kcal} / 100 \mathrm{~g})\end{array}$ \\
\hline Masa cruda & & $48.99 \pm 4.79^{\mathrm{a}}$ & $25.21 \pm 0.15^{\mathrm{a}}$ & $9.89 \pm 1.42^{\mathrm{a}}$ & $1.65 \pm 0.02^{\mathrm{b}}$ & $7.63 \pm 0.12^{\mathrm{a}}$ & $385.13 \pm 12.44^{\mathrm{a}}$ \\
\hline Bollo cocido & & $45.89 \pm 3.22^{\mathrm{a}}$ & $29.84 \pm 1.07^{\mathrm{b}}$ & $9.65 \pm 0.77^{\mathrm{a}}$ & $0.48 \pm 0.05^{\mathrm{c}}$ & $7.59 \pm 0.29^{\mathrm{a}}$ & $373.36 \pm 9.36^{\mathrm{a}}$ \\
\hline
\end{tabular}

Tabla 2. Composición química de la masa y el bollo en 100 g de muestra

*Los datos representan la media de tres determinaciones, con su respectiva desviación estándar. Superíndices diferentes en una misma columna indican diferencias significativas $(p<0.05)$. Fuente: Autores

En la tabla 3 se presentan las propiedades termofísicas del bollo de maíz cariaco amarillo obtenido a las diferentes temperaturas durante la cocción. 
Tabla 3. Propiedades termofísicas del bollo de maíz cariaco amarillo en función de la temperatura

\begin{tabular}{|l|l|c|c|}
\hline \multirow{2}{*}{ Parámetros } & \multirow{2}{*}{ Unidades } & $20^{\circ} \mathrm{C}$ & $98^{\circ} \mathrm{C}$ \\
\cline { 3 - 4 } & & Masa* $^{*}$ & Bollos* \\
\hline Conductividad térmica & $\mathrm{W} / \mathrm{m}^{\circ} \mathrm{C}$ & $0.51 \pm 0.03^{\mathrm{a}}$ & $0.68 \pm 0.07^{\mathrm{b}}$ \\
\hline Densidad & $\mathrm{kg} / \mathrm{m}^{3}$ & $1233.08 \pm 45.36^{\mathrm{a}}$ & $1254.49 \pm 30.47^{\mathrm{a}}$ \\
\hline Capacidad calorífica & $\mathrm{J} / \mathrm{Kg}^{\circ} \mathrm{C}$ & $3132.63 \pm 87.84^{\mathrm{a}}$ & $3145.36 \pm 88.45^{\mathrm{a}}$ \\
\hline Difusividad térmica & $\mathrm{m}^{2} / \mathrm{s}$ & $1.33 \pm 0.01^{\mathrm{a}} \times 10^{-7}$ & $1.98 \pm 0.02^{\mathrm{b}} \times 10^{-7}$ \\
\hline
\end{tabular}

*Los datos representan la media de tres determinaciones con su respectiva desviación estándar. Superíndices diferentes en una misma fila indican diferencias significativas $(\mathrm{p}<0.05)$. Fuente: autores

\section{Perfiles de tiempo y temperatura del bollo de maíz}

Se puede observar en la figura 2, el historial térmico de los bollos de maíz durante el proceso de cocción por ebullición. Mientras que en la figura 3, se muestra la linealización de la relación adimensional de la temperatura vs tiempo, lo cual permitió calcular la pendiente y con esta determinar el coeficiente $h$.

Figura 2. Perfiles térmico del bollo de maíz durante el proceso de cocción

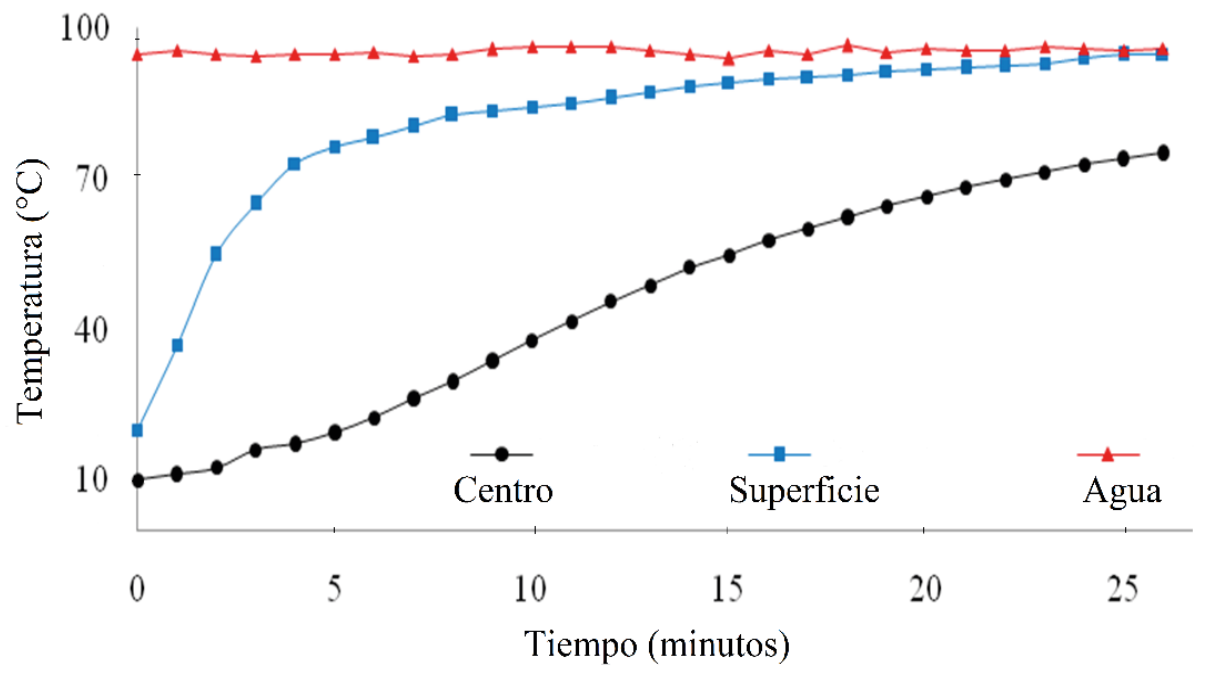

Fuente: Autores 
Figura 3. Relación adimensional de la temperatura vs el tiempo

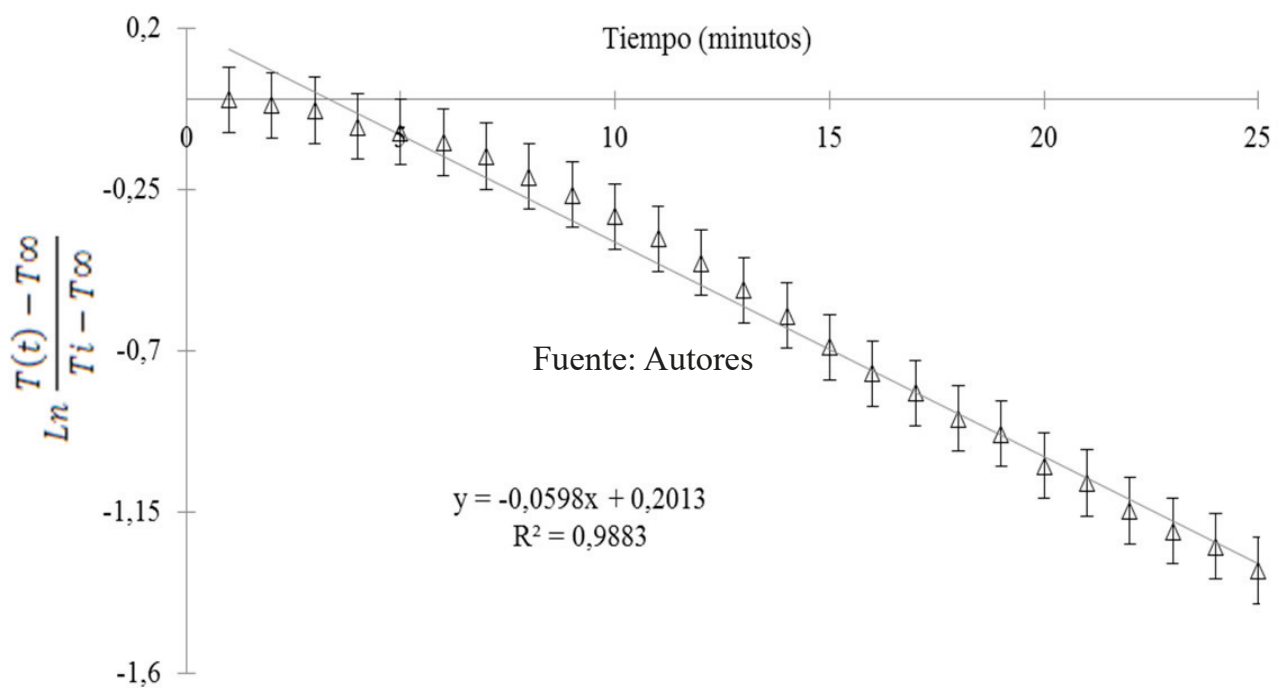

En la tabla 4, se muestra el coeficiente de transferencia de calor obtenido mediante el análisis de regresión lineal durante el proceso de cocción por ebullición del bollo de maíz. Así mismo, se presenta el valor conseguido del número de biot y el coeficiente de determinación ajustado.

Tabla 4. Valores del coeficiente de transferencia de calor $(h)$ de bollos de maíz

\begin{tabular}{|c|c|c|c|c|}
\hline & $\begin{array}{c}\text { Pendiente } \\
(\mathrm{m})\end{array}$ & $\begin{array}{c}\text { Número de } \\
\text { Biot }\end{array}$ & $\begin{array}{c}\text { Coeficiente convectivo de transferencia de } \\
\text { calor }(h) \mathrm{W} / \mathrm{m}^{2 \circ} \mathbf{C}\end{array}$ & $\begin{array}{c}\text { Correlación } \\
\left(\mathrm{R}^{2}\right)\end{array}$ \\
\hline & -0.059 & 2.65 & 118.29 & 0.99 \\
\hline
\end{tabular}

Fuente: Autores

\section{Discusión de resulatdos}

\section{Composición química}

Se observó que el contenido de humedad fue más alto en el producto final $(29.84 \pm 1.07)$ respecto a la masa antes de procesamiento $(\mathrm{p}<0.05)$, esta diferencia se puede explicar por la cantidad de agua que ingresa al alimento durante la cocción por ebullición (Ayadi et al., 2009). También se encontró que el contenido de cenizas fue significativamente menor en los bollos cocidos (0.48 \pm 0.05$)$, lo que se atribuyó a que los minerales quedaron retenidos en el agua del proceso (Carciofi et al., 2002; Iborra $\square$ Bernad et al., 2014). En el contenido de grasa no se observaron diferencias estadísticamente significativas ( $\mathrm{p}>0.05$ ) mostrando para ambos casos valores alrededor del $6 \%$, siendo estos promedios bajos al comparar con otros productos similares, lo cual puede ser debido a que la harina utilizada e durante la elaboración de los bollos era desengrasada (Arámbula-Villa et al., 2001; Gamboa, 2012; Torres et al., 2016a). En 
general el producto cocido mostró porcentajes más bajos de carbohidratos, fibra bruta y proteínas $(45.89 \pm 3.22$, $9.65 \pm 0.77$ y $7.59 \pm 0.29$ respectivamente) debido a que estas son sustancias hidrofílica y posiblemente quedaron acumuladas en el medio de acuoso; sin embargo, no hubo diferencias estadísticas entre los promedios según el ANOVA ( $p>0.05)$. En el caso de la fibra es importante destacar que dichos valores fueron similares a los reportados por Valenzuela, Ortiz y Pérez (2013) en productos de maíz blanco, estos autores comentan a su vez las ventajas de la fibra en este tipo de alimentos, dado sus beneficios en el funcionamiento del sistema digestivo. Por un lado, el aporte de fibra puede tener efectos positivos en los procesos de control del estreñimiento, regulación de la glucemia, protección contra el cáncer de colon y menor riesgo de enfermedades cardiovasculares. Las calorías calculadas para el bollo de maíz estuvo dentro de lo reportado para alimentos amiláceos similares y procesados térmicamente (Alvis, González y Arrázola, 2015 y Torres et al., 2016a) además entre los datos de la masa y el maíz el ANOVA no detectó diferencias estadísticamente significativas ( $p>0.05)$. Con relación a lo anterior, se evidencia que los bollos de maíz constituyen una fuente importante de macronutrientes, por lo cual, a partir de los resultados encontrados se recomienda el consumo frecuente de este producto, especialmente por aquellas personas que carecen de estos componentes esenciales en la nutrición y es posible catalogarlo como un alimento nutricionalmente completo.

\section{Análisis de las propiedades termofisicas}

Los valores de las propiedades termofísicas obtenidos en el bollo de maíz teniendo en cuenta la composición químicas y la temperatura de procesamiento fueron para la conductividad térmica, densidad, capacidad calorífica y difusividad térmica de $0.68 \pm 0,07 \mathrm{~W} / \mathrm{m}^{\circ} \mathrm{C}, 1254.49$ $\pm 30.47 \mathrm{~kg} / \mathrm{m}^{3}, 3145.36 \pm 88.45 \mathrm{~J} / \mathrm{kg}^{\circ} \mathrm{C}$ y $1.98 \pm 0.02 \mathrm{x}$ $10^{-7} \mathrm{~m}^{2} / \mathrm{s}$ respectivamente. Se presentaron diferencias estadísticamente significativas respecto a la masa cruda, solo en los parámetros $k$ y $\alpha(\mathrm{p}<0,05)$. Estos resultados encontrados para los bollos de maíz, coinciden con los reportados para otros alimentos de origen vegetal. Rodríguez, Salamanca y Abril (2008) encontraron para cinco alimentos colombianos (lechona, tamal, envuelto de mute, bollo de yuca y subido de maíz), valores para la conductividad $\left(0.49,0.52,0.55,0.51\right.$ y $\left.0.54 \mathrm{~W} / \mathrm{m}^{\circ} \mathrm{C}\right)$ difusividad $\left(1.42 \times 10^{-7}, 1.47 \times 10^{-7}, 1.51 \times 10^{-7}, 1.50 \times 10^{-7} \mathrm{y}\right.$ $\left.1.49 \times 10^{-7} \mathrm{~m}^{2} / \mathrm{s}\right)$ capacidad calorífica $(3398,3479,3396$, 3348 y $\left.3357 \mathrm{~J} / \mathrm{Kg}{ }^{\circ} \mathrm{C}\right)$ y para la densidad $(1055,8 ; 1027,5$; $1081,40,1093,86$ y $1081,52 \mathrm{~kg} / \mathrm{m}^{3}$ ) respectivamente.
Para Moraga, Zambra, Torres y Lemus-Mondaca (2011) quienes determinaron las propiedades termofísicas para durazno, cereza e uva, encontraron valores para la densidad $\left(1038.60,1064.11,1070.16 \mathrm{~kg} / \mathrm{m}^{3}\right), \mathrm{Cp}$ $\left(3893.75,3753.43, \quad 3671.66 \mathrm{~J} / \mathrm{kg}^{\circ} \mathrm{C}\right)$, conductividad térmica $\left(0.57,0.56,0.55 \mathrm{~W} / \mathrm{m}^{\circ} \mathrm{C}\right)$ respectivamente. Así mismo, Verlinden, Nicolai y De Baerdemaeker (1995) determinaron estos valores en cilindros de papas y obtuvieron valores para la conductividad térmica $(0.65$ $\left.\mathrm{W} / \mathrm{m}^{\circ} \mathrm{C}\right)$, densidad $\left(1085 \mathrm{~kg} / \mathrm{m}^{3}\right)$, capacidad calorífica $\left(3610 \mathrm{~J} / \mathrm{kg}^{\circ} \mathrm{C}\right)$. Por su parte, En esta misma tendencia Carciofi et al. (2002), Determinaron la difusividad térmica en la cocción por inmersión de mortadela, a partir de datos de procesamiento y reportaron valores mínimos de $2.42 \times 10^{-7} \mathrm{~m}^{2} / \mathrm{s}$. Arrazola, Páez y Alvis (2014) en su trabajo la difusividad, conductividad térmica, calor específico y densidad de almendras frescas fueron de $1.13 \times 10^{-7} \mathrm{~m}^{2} / \mathrm{s}, 0.32 \mathrm{~W} / \mathrm{m}^{\circ} \mathrm{C}, 2.65 \mathrm{~kJ} / \mathrm{kg}{ }^{\circ} \mathrm{C}$ y $1138.6 \mathrm{~kg} /$ $\mathrm{m}^{3}$ respectivamente. Yıldız, Palazoğlu y Erdoğdu (2007) en trozos de papa, obtuvieron valores de conductividad térmica de $0.40 \mathrm{~W} / \mathrm{m}^{\circ} \mathrm{C}$ a $0.60 \mathrm{~W} / \mathrm{m}^{\circ} \mathrm{C}$, mientras que la difusividad térmica estuvo entre $9.2 \times 10^{-9}, 11.2 \times 10^{-9} \mathrm{y}$ $18.2 \times 10^{-9} \mathrm{~m}^{2} / \mathrm{s}$ a las temperaturas de 150,170 y $190^{\circ} \mathrm{C}$. Por su parte Alvis, Cortés y Páez (2009) indicaron que la conductividad y la difusividad térmica fueron de 0.44 $\mathrm{W} / \mathrm{m}{ }^{\circ} \mathrm{C}$ y $1.23 \times 10^{-7} \mathrm{~m}^{2} / \mathrm{s}$, en trozos de ñame sometidos a cocción en aceite a temperaturas entre 140 y $180^{\circ} \mathrm{C}$. Algunos investigadores han señalado que para los alimentos amiláceos, la conductividad térmica disminuye en proporción con la mayor cantidad de aire en el interior de los poros y células (Kanjanapongkul, 2017).

Teniendo en cuenta esto, posiblemente a las condiciones de temperatura utilizadas, hubo más reducción del aire en la microestructura de los bollos de maíz cocidos, lo que provocaría un aumento de esta propiedad. Baik y Mittal (2003) encontraron que el calor específico varió de 2.52 a $3.69 \mathrm{~kJ} \mathrm{~kg}^{-1} \mathrm{~K}^{-1}$ con rangos de temperaturas entre $10-105{ }^{\circ} \mathrm{C}$. Mientras que las demás propiedades térmicas del tofu (producto a base de soya) a intervalos de $5-80{ }^{\circ} \mathrm{C}$ y rangos de humedad entre 0.3 a 0.7 (base húmeda). Fueron, $k$ : 0.24 a $0.43 \mathrm{~W} \mathrm{~m}^{-1} \mathrm{~K}^{-1}$ y $\alpha: 7.6 \mathrm{x}$ $10^{-8} \mathrm{a}-1.15 \times 10^{-7} \mathrm{~m}^{2} \mathrm{~s}^{-1}$. En dicha investigación los modelos empíricos simples se desarrollaron como una función del contenido de humedad y la temperatura. Los resultados encontrados en esta investigación para bollos de maíz fueron similares a los hallados por Kim, Lim, Bae, Lee y Lee (2011) quienes indicaron una $k$ igual a $0.55 \mathrm{~W} / \mathrm{m}{ }^{\circ} \mathrm{C}$ y $\alpha=1.45 \times 10^{-7} \mathrm{~m}^{2} / \mathrm{s}$ en trozos de papa cocidos en aceite a $170{ }^{\circ} \mathrm{C}$. Mientras que para Farinu y 
Baik (2007) la conductividad fue $0.49 \pm 0.04 \mathrm{Wm}^{-1} \mathrm{~K}^{-1}$, el calor específico de $3660 \pm 477.41 \mathrm{Jkg}^{-1} \mathrm{~K}^{-1}$ y la densidad $1.21 \pm 73.5 \mathrm{~kg} / \mathrm{m}^{3}$. Recientemente Alvis et al. (2015), determinaron las propiedades termofísicas en chips de batatas y encontraron en el caso de los productos no recubiertos (NR) con biopolímeros, que la conductividad térmica varió de 0.39 a $0.45 \mathrm{~W} / \mathrm{m}^{\circ} \mathrm{C}$, mientras que en los recubrimientos (CR), los valores estuvieron entre 0.41 y $0.46 \mathrm{~W} / \mathrm{m}^{\circ} \mathrm{C}$. Por su parte, el calor específico estuvo entre 2470 a $2920 \mathrm{~kJ} / \mathrm{kg}{ }^{\circ} \mathrm{C}$ y 2500 a $2960 \mathrm{~kJ} / \mathrm{kg} \mathrm{K}$, mientras que la difusividad térmica fue de 1.16 a 1.35 x $10^{7} \mathrm{~m}^{2} / \mathrm{s}$, para NR y de 1.19 a $1.34 \times 10^{-7} \mathrm{~m}^{2} / \mathrm{s}$ en CR. En dicha investigación la variación de la densidad estuvo entre 1150 a $2920 \mathrm{~kg} / \mathrm{m}^{3}$ y 1150 a $2960 \mathrm{~kg} / \mathrm{m}^{3}$ para NR y $\mathrm{CR}$ respectivamente, siendo estos valores relativamente más bajos que los hallados en los bollos de maíz. Siendo estas las diferencias atribuidas a las variabilidades en la composición química y microestructura de estas matrices alimentarias.

\section{Perfil térmico y coeficiente convectivo de transferencia de calor}

El centro térmico del producto registrado con los termopares presentó un incremento de temperatura lineal, llegando hasta los $75^{\circ} \mathrm{C}$ durante 25 minutos (condiciones adecuadas de cocción del producto). Se observó que en la superficie de los bollos, la temperatura aumentó rápidamente al comienzo del proceso (10 minutos) estabilizándose en $82 \pm 1^{\circ} \mathrm{C}$ y llegando a los $90{ }^{\circ} \mathrm{C}$ a partir de los 20 minutos. Estas graficas son importantes ya que reflejan el perfil térmico en el interior de este alimento durante la cocción por ebullición; por lo tanto, pueden ayudar a definir las condiciones de tiempo y temperatura óptimas para este proceso, así mismo pueden servir de base para futuros trabajos de investigación donde se analicen tratamientos térmicos similares en este producto como la esterilización, cocción al vapor, al vacío y en aceite etc. El valor de coeficiente de transferencia de calor encontrado para el bollo de maíz cocido fue de $118.29 \mathrm{~W} / \mathrm{m}^{2}{ }^{\circ} \mathrm{C}$, fue similar a los obtenidos por Awuah, Ramaswamy y Simpson (1993) quienes estudiaron el proceso de cocción por inmersión en agua para cilindros de papas y zanahorias recubiertos con carboximetilcelulosa (CMC) y reportaron valores $h$ entre 150 y $165 \mathrm{~W} / \mathrm{m}^{2}{ }^{\circ} \mathrm{C}$ y $80-456 \mathrm{~W} / \mathrm{m}^{2}{ }^{\circ} \mathrm{C}$ respectivamente, con temperaturas entre 50 y $100{ }^{\circ} \mathrm{C}$. Por su parte, Verlinden et al. (1995), estudiaron el proceso de gelatinización del almidón en cilindros de papa y determinaron el valor de $h$ adecuado de $750 \mathrm{~W} / \mathrm{m}^{2 \circ} \mathrm{C}$ con una temperatura del fluido a $100^{\circ} \mathrm{C}$, siendo este relativamente más alto que el hallado para los bollos de maíz. Costa y Oliveira (1999) en el proceso de cocción de papas en aceite reportaron valores de $h$ entre 140 y $165 \mathrm{~W} / \mathrm{m}^{2 \circ} \mathrm{C}$, para temperaturas entre $60 \mathrm{y}$ $100{ }^{\circ} \mathrm{C}$. Similarmente Sahin, Sastry y Bayindirli (1999) determinaron el coeficiente de transferencia de calor transitoria de un paralelepípedo de aluminio relleno con masa de papa y sumergido en aceite de girasol a las temperaturas de 150 y $190{ }^{\circ} \mathrm{C}$, encontraron valores de $h$ entre 90 y $200 \mathrm{~W} / \mathrm{m}^{2}{ }^{\circ} \mathrm{C}$. Así mismo, Farkas y Hubbard (2000) usaron cilindros de papa para determinar el coeficiente $h$ a $180^{\circ} \mathrm{C}$ y encontraron que para un tiempo de 200 a 900 segundos en la fase de ebullición, el valor de este parámetro se ubicó entre 300 y $1100 \mathrm{~W} / \mathrm{m}^{2}{ }^{\circ} \mathrm{C}$.

Vélez-Ruiz y Sosa-Morales (2003) usaron donas de forma cilíndrica con anillo interior de $1.5 \mathrm{~cm}$ y anillo exterior $2.0 \mathrm{~cm}$ y determinaron $h$ durante la cocción en aceite a temperaturas de 180,190 y $200{ }^{\circ} \mathrm{C}$, indicaron valores de $158.63,269.54$ y $296.84 \mathrm{~W} / \mathrm{m}^{2}{ }^{\circ} \mathrm{C}$ respectivamente. Budzaki y Seruga (2005) encontraron que $h$ estuvo entre $94.22 \pm 0.33$ y $774.88 \pm 3.89 \mathrm{~W} / \mathrm{m}^{2}{ }^{\circ} \mathrm{C}$ para esferas de harina de trigo y papa (50:50) procesadas en aceite a 190 ${ }^{\circ} \mathrm{C}$, estos autores mostraron que los valores más altos de los coeficientes convectivos, se observaron en la etapa de ebullición. Por otro lado, Yildiz et al. (2007), encontraron una disminución de los coeficientes convectivos de transferencia de calor con el aumento de la temperatura, reportando valores de $286.7,227.3$ y $181.3 \mathrm{~W} / \mathrm{m}^{2}{ }^{\circ} \mathrm{C}$ para temperaturas de 150,170 y $190{ }^{\circ} \mathrm{C}$ respectivamente. Así mismo, Farinu y Baik (2007) trabajaron con esferas de batata sometidas a temperaturas de $150,160,170$ y 180 ${ }^{\circ} \mathrm{C}$ y encontraron valores de $h$ entre 710 y $837 \mathrm{~W} / \mathrm{m}^{2}{ }^{\circ} \mathrm{C}$. Por su parte, Alvis et al. (2009), en la cocción de trozos de ñames en aceite de girasol encontraron valores de $h$ entre 148.10 y 204.46 para temperaturas entre 140 y $180^{\circ} \mathrm{C}$.

Recientemente, Franklin, Pushpadass, RavindraMenon, Rao y Nath (2014) encontraron valores de $h$ entre $90-130 \mathrm{~W} / \mathrm{m}^{2}{ }^{\circ} \mathrm{C}$ para la cocción en aceite de gulabjamun (postre típico de la india). Mientras que para Alvis et al. (2015), el coeficiente convectivo fue mayor cuando aumentó la temperatura de cocción del aceite y se utilizó recubrimiento comestible. Los valores obtenidos fueron de $144.22,168.15$ y $199.82 \mathrm{~W} / \mathrm{m}^{2}{ }^{\circ} \mathrm{C}$ para las muestras sin recubrimiento a temperaturas de 150,170 y $190{ }^{\circ} \mathrm{C}$ respectivamente y de $188.75,228.41$ y $239.06 \mathrm{~W} / \mathrm{m}^{2}$ ${ }^{\circ} \mathrm{C}$ para las mismas temperaturas. Neethu et al. (2015), indicaron que cuando la temperatura de cocción en aceite aumentó de 125 a $145^{\circ} \mathrm{C}$, el coeficiente de transferencia 
de calor obtenido para la pantoa (producto lácteo de la india) se incrementó de 101.77 a $237.10 \mathrm{~W} / \mathrm{m}^{2}{ }^{\circ} \mathrm{C}$. Por su parte, Mondal y Dash (2016) utilizando el análisis de los sistemas concentrados, modelaron la transferencia de calor durante la cocción en aceite de Chhena Jhili (postre a base de trigo típico del sur de india) y encontraron que los coeficientes convectivos $h$ estuvieron en el intervalo de 43.59 y $52.92 \mathrm{~W} / \mathrm{m}^{2}{ }^{\circ} \mathrm{C}$, con números de Biot en el intervalo de 1.07-1.32 respectivamente. Alternativamente también resolvieron con otro enfoque, la ecuación de conducción de calor transitorio y encontraron que los parámetros de transferencia de calor a temperaturas entre 125 y $145^{\circ} \mathrm{C}$ fueron: $k=0.42$ y $0.43 \mathrm{~W} / \mathrm{m}^{\circ} \mathrm{C}, \alpha=1.29$ $\mathrm{x} 10^{-5}$ a $1.32 \times 10^{-5} \mathrm{~m}^{2} / \mathrm{s}, h=103.19$ y $69.81 \mathrm{~W} / \mathrm{m}^{2}{ }^{\circ} \mathrm{C}$, con números de biot $(\mathrm{Bi})$ entre 3.85 y 2.56 . En general estos autores señalaron que los valores estimados estuvieron en el rango reportado para diferentes productos alimenticios con diversas geometrías. Estos coeficiente fueron más bajos que los encontrados por Sandhu et al. (2016), durante una simulación de cocción en aceite a temperaturas de 150,170 y $190^{\circ} \mathrm{C}$, donde los valores máximos fueron de 3617,4517 y $7307 \mathrm{~W} / \mathrm{m}^{2}{ }^{\circ} \mathrm{C}$ respectivamente. Por otro lado, Erim Kose y Dogan (2016) determinaron el coeficiente de $h$ en la cocción en aceite de Tulumba (postre típico de Turquía) y encontraron que a mayor temperatura disminuyó $h$, el cual tuvo un valor máximo de $182.41 \mathrm{~W} / \mathrm{m}^{2}{ }^{\circ} \mathrm{C}$ a $150^{\circ} \mathrm{C}$ y un mínimo de $125.72 \mathrm{~W} / \mathrm{m}^{2}$ ${ }^{\circ} \mathrm{C}$ a $180^{\circ} \mathrm{C}$. En general las diferencias entre los valores del coeficiente de transferencia de calor encontrados en el presente trabajo para la cocción de bollos de maíz y los reportados en la literatura, pueden ser explicadas por la diferencia entre las microestructuras, las geometrías de los productos, el contenido de humedad inicial, las técnicas utilizados para las mediciones de las temperaturas y los métodos de solución de las ecuaciones.

\section{Conclusiones}

El tratamiento térmico (tiempo-temperatura) ideal durante la cocción atmosférica para muestras de cilindros de bollo de maíz fue de 25 minutos hasta una temperatura $75^{\circ} \mathrm{C}$ en el centro del producto. Los valores de la conductividad y difusividad térmica aumentaron linealmente con la temperatura de procesamiento $(p<0,05)$, y estuvieron dentro de lo reportado por otros autores para productos amiláceos de naturaleza similar. El coeficiente convectivo $h$ fue de $118.287 \mathrm{~W} / \mathrm{m}^{2} \mathrm{~K}$ y los valores obtenidos de las propiedades termofísicas en función de la composición química y la temperatura fueron para la conductividad $0.68 \pm 0.07 \mathrm{~W} / \mathrm{m}{ }^{\circ} \mathrm{C}$, difusividad $1.98 \pm 0.02 \times 10^{-7} \mathrm{~m}^{2} / \mathrm{s}$ y la capacidad calorífica de $3145.36 \pm 88.45 \mathrm{~J} / \mathrm{kg}{ }^{\circ} \mathrm{C}$. El entendimiento de los parámetros de transferencia de calor durante la cocción de bollo de maíz es importante para la optimización de procesos térmicos aplicados a esta matriz alimentaria.

Nomenclatura: $h=$ Coeficiente de transferencia de calor $\left(W / m^{2 \circ} \mathrm{C}\right) ; k=$ conductividad $\left(W m^{-1}{ }^{\circ} \mathrm{C}{ }^{-1}\right) ; t=$ Tiempo (s); $m=$ Masa (Kg); $T=$ Temperatura $\left({ }^{\circ} \mathrm{C}\right)$; $\Delta T=$ Diferencia de temperatura $\left({ }^{\circ} \mathrm{C}\right) ; T_{\infty}=$ Temperatura del agua de cocción $\left({ }^{\circ} \mathrm{C}\right) ; T_{o}=$ Temperatura inicial $\left({ }^{\circ} \mathrm{C}\right)$; $T=$ Temperatura localizada en un punto a un tiempo $t\left({ }^{\circ} \mathrm{C}\right)$; $\Delta h=$ cambio de entalpia $(\mathrm{kJ} / \mathrm{Kg}) ; A=$ Área de superficie $\left(\mathrm{m}^{2}\right) ; \rho=$ Densidad $\left(\mathrm{kg} / \mathrm{m}^{3}\right) ; C_{p}=$ Calor especifico $(\mathrm{kJ} /$ $\left.\mathrm{kg}{ }^{\circ} \mathrm{C}\right) ; V=$ Volumen del bollo de maiz $\left(\mathrm{m}^{3}\right) ; Q=$ flujo de energía en forma de calor $(J)$.

Descargos de responsabilidad: El manuscrito fue preparado y revisado con la participación de todos los autores, quienes declaramos que no existe ningún conflicto de intereses que ponga en riesgo los resultados presentados.

\section{Referencias}

Alvis, A., Caicedo, I. y Peña, P. (2012). Determinación de Propiedades Termofísicas de Alimentos en Función de la Concentración y la Temperatura empleando un Programa Computacional. Información tecnológica, 23(1): 111-116.

Alvis, A., Cortés, L. E., \& Páez, M. (2009). Transferencia de Calor y Materia durante la Fritura de Trozos de Ñame (Dioscórea alata). Información tecnológica, 20(1): 99-109.

Alvis, A., González, A, y Arrázola, G. (2015). Efecto del Recubrimiento Comestible en las Propiedades de Trozos de Batata (Ipomoea Batatas Lam) Fritos por Inmersión: Parte 2: Propiedades Termofísicas y de Transporte. Información tecnológica, 26(1): 103-116. 
AOAC (2012). Official Methods of Analysis International. International. Washington D.C.

Arámbula-Villa, G., Barron-Avila, L., Gonzalez-Hernández, J., Moreno-Martinez, E. y Luna-Barcenas, G. (2001). Efecto del tiempo de cocimiento y reposo del grano de marz (Zea mays L.) nixtamalizado, sobre las caracteristicas fisicoquimicas, estructurales y reológicas, estructurales y texturales, masa y tortilla de ma1z. Archivo Latinoamericanos de Nutrición, 51(2): 187-194.

Arrazola, G., Páez, M. y Alvis A. (2014). Composición, Análisis termofísico y análisis sensorial de frutos colombianos: Parte 1: Almendro (Terminalia Catappa L.). Información Tecnológica, 25(3), 17-22.

Awuah, GB., Ramaswamy, HS, y Simpson, BK. (1993). Surface heat transfer coefficients associated with heating of food particles in CMC solutions. Journal of food process engineering, 16(1): 39-57.

Ayadi, M. A., Makni, I. y Attia, H. (2009). Thermal diffusivities and influence of cooking time on textural, microbiological and sensory characteristics of turkey meat prepared products. Food and Bioproducts Processing, 87(4): 327-333.

Baik,ODyMittal,GS.(2003). Determinationandmodeling of thermal properties of tofu. International Journal of Food Properties, 6(1): 9-24.

Bouasla, A., Wójtowicz, A., \& Zidoune, M. N. (2017). Gluten-free precooked rice pasta enriched with legumes flours: Physical properties, texture, sensory attributes and microstructure. LWT-Food Science and Technology, 75: 569-577.

Budžaki, S. y Šeruga, B. (2005). Moisture loss and oil uptake during deep-fat frying of "Kroštula" dough. European Food Research and Technology, 220(1): 90-95.

Carciofi, B. A., Faistel, J., Aragão, G. M. y Laurindo, J. B. (2002). Determination of thermal diffusivity of mortadella using actual cooking process data. Journal of Food Engineering, 55(1): 89-94.
Castilla, Y., Mercado, I., Mendoza, V., Monroy M. (2011). Determinación y cuantificación de los niveles de aflatoxinas en bollos de mazorca producidos en Arjona (Bolívar - Colombia). Avances Investigaciones en Ingeniería, 8(1): 69-74.

Costa, RM, y Oliveira, FA. (1999). Modelling the kinetics of water loss during potato frying with a compartmental dynamic model. Journal of Food Engineering, 41(3): 177-185.

Erim Kose, Y. y Dogan, I. S. (2016). Determination of Simultaneous Heat and Mass Transfer Parameters of Tulumba Dessert during Deep $\square$ Fat Frying. Journal of Food Processing and Preservation. 00. 00.

Farinu, A. y O.D. Baik (2007) Heat transfer coefficients during deep fat frying of sweetpotato: Effects of product size and oil temperature, Food Research International: 40: 989-994.

Farkas, B. E. y L.J. Hubbard (2000). Analysis of convective heat transfer during immersion frying drying. Journal of Food Technology, 18(5): $145-151$.

Franklin, M. E. E., Pushpadass, H. A., RavindraMenon, R., Rao, K. J., y Nath, B. S. (2014). Modeling the heat and mass transfer during frying of gulab jamun. Journal of Food Processing and Preservation, 38(4), 1939-1947.

Gamboa, L., García, M. y Tablante, L. (2012). Evaluación nutricional y sensorial de arepas a base de harinas de maíz blanco (zea mays 1.) yuca dulce (Manihot esculenta Crantz) enriquecidas con texturizado de proteína de soya (Glycine max). Saber. Revista Multidisciplinaria del Consejo de Investigación de la Universidad de Oriente, 24(2), 185-190.

Iborra-Bernad, C., García-Segovia, P. y Martínez-Monzó, J. (2014). Effect of vacuum cooking treatment on physicochemical and structural characteristics of purple $\square$ flesh potato. International Journal of Food Science \& Technology, 49(4), 943-951. 
Kanjanapongkul, K. (2017). Rice cooking using ohmic heating: Determination of electrical conductivity, water diffusion and cooking energy. Journal of Food Engineering, 192: 1-10.

Kim, D. N., Lim, J., Bae, I. Y., Lee, H. G., \& Lee, S. (2011). Effect of hydrocolloid coatings on the heat transfer and oil uptake during frying of potato strips. Journal of Food Engineering, 102(4): 317-320.

Mondal, I. H. y Dash, K. K. (2016). Textural, Color Kinetics, and Heat and Mass Transfer Modeling During Deep Fat Frying of Chhena Jhili. Journal of Food Processing and Preservation, 00:00.

Moraga, N. O., Zambra, C. E., Torres, P. B. y Lemus-Mondaca, R. A. (2011). Modelado de dinámica de fluidos y transferencia de calor $\mathrm{y}$ masa en procesos agroalimentarios por método de volúmenes finitos. Dyna, 78(169), 140-149.

Murador, D. C., Mercadante, A. Z., y de Rosso, V. (2016). Cooking techniques improve the levels of bioactive compounds and antioxidant activity in kale and red cabbage. Food chemistry, 196: 1101-1107.

Neethu, K. C., Franklin, M. E., Pushpadass, H. A., Menon, R. R., Rao, K. J. y Nath, B. S. (2015). Analysis of Transient Heat and Mass Transfer during Deep $\square$ Fat Frying of Pantoa. Journal of Food Processing and Preservation, 39(6): 966-977.

Palermo, M., Pellegrini, N., \& Fogliano, V. (2014). The effect of cooking on the phytochemical content of vegetables. Journal of the Science of Food and Agriculture, 94(6), 1057-1070.

Rodríguez, O., G. Salamanca y J.Abril(2008). Simulación y optimización de un proceso de transferencia de calor para la inactivación de clostridium botulinum en alimentos colombianos Ibagué. Universidad del Tolima. Facultad de Ciencias de la Universidad del Tolima. 1-4.

Sahin, S., S.K. Sastry y L. y Bayindirli (1999) Heat transfer during frying of potato slices, Journal of Food Engineering, 32(1): 19-24.
Sandhu, J., Parikh, A., \& Takhar, P. S. (2016). Experimental determination of convective heat transfer coefficient during controlled frying of potato discs. LWT-Food Science and Technology, 65: 180-184.

Siripon, K., Tansakul, A., \& Mittal, G. S. (2007). Heat transfer modeling of chicken cooking in hot water. Food Research International, 40(7): 923-930.

Torrenegra, M., Granados, C., Acevedo, D., Guzmán, L., Álvarez, I, Padilla N. (2013). Caracterización del proceso de elaboración del bollo limpio y de mazorca en Villa-Nueva, MUNICIPIO DEL departamento. Bolívar-Colombia. Revista Biotecnología en el Sector Agropecuario y Agroindustrial. 11(2): 148-155.

Torres, JD., Acevedo, D. y Tirado DF. (2015). Análisis de la calidad bromatológica, microbiológica, sensorial y textura de bollo de mazorca cocidos en ebullición. Revista Reciteia. 14(2): 7-16.

Torres, JD., Acevedo, D. y Montero, P. M. (2016b). Proyectos de Aula Semestrales como Estrategia Pedagógica para la Formación en Ingeniería. Formación universitaria, 9(3): 23-30.

Torres, JD., Acevedo, D. y Montero, P. M. (2016a). Influencia del almacenamiento en la textura y viscoelasticidad de bollos de maíz Cariaco blanco. Corpoica Ciencia y Tecnología Agropecuaria, 17(3): 403-416.

Valenzuela, LA., Ortiz, B. L. y Pérez, C. E. (2013). Estudio comparativo del efecto metabólico de arepas enriquecidas con pectina extraída de guayaba (Psidium guajava L.) o pectina cítrica comercial. Revista Colombiana de Química, 42(3): 302-325.

Vélez-Ruíz， J.F. y Sosa-Morales, M.E. (2003). Evaluation of physical properties of dough of donuts during deep-fat frying ay different temperaturas. Internacional Journal of Food Properties, 6(2): 341-353. 